\title{
An unusual association between Mycobacterium tuberculosis and Aspergillus fumigatus
}

\author{
R. Agarwal, N. Singh, A.N. Aggarwal
}

ABSTRACT: An unusual association between Mycobacterium tuberculosis and Aspergillus fumigatus. R. Agarwal, N. Singh, A.N. Aggarwal.

Allergic bronchopulmonary aspergillosis (ABPA) is rarely described outside the setting of asthma or cystic fibrosis. The occurrence of ABPA in other structural lung diseases included scars of old healed pulmonary tuberculosis (PTB) is also unknown. In this case, we report a 62year old lady treated for PTB 40 years ago who presented with increasing dyspnea on exertion, cough with expectoration of blackish brown mucus plugs and wheezing.

\begin{abstract}
High-resolution computed tomographic scan of the thorax showed parenchymal fibrosis and volume loss in left upper lobe while central bronchiectasis, mosaic attenuation, centrilobular nodules with a tree-in-bud pattern were observed in the other lobes. Investigations revealed a diagnosis of ABPA. The patient was treated with prednisolone and showed a significant response. We review the current literature on this unusual association of previous and cured TB with ABPA, and also discuss the hypothesis of this possible relationship.
\end{abstract}

Monaldi Arch Chest Dis 2008; 69: 1, 32-34.

Keywords: Allergic bronchopulmonary aspergillosis, ABPA, Pulmonary tuberculosis, Aspergillus, Mycobacterium.

Department of Pulmonary Medicine, Post Graduate Institute of Medical Education and Research (PGIMER), Chandigarh, India.

Correspondence: Dr. Ritesh Agarwal, Assistant Professor, Department of Pulmonary Medicine, Post Graduate Institute of Medical Education and Research, Sector 12, Chandigarh-160012, India; e-mail: riteshpgi@gmail.com; ritesh@indiachest.org

\section{Introduction}

Allergic bronchopulmonary aspergillosis (ABPA) commonly occurs in the setting of bronchial asthma or cystic fibrosis. Unusually, ABPA has been reported to complicate chronic granulomatous disease and hyper-IgE syndrome [1]. Its prevalence in patients with other disorders especially structural lung disease is unknown and its occurrence in patients with past history of pulmonary tuberculosis (PTB) is also extremely rare [2]. The current case describes the occurrence of ABPA in a 62 year old lady who was treated for PTB 40 years ago. We also describe the possible mechanisms that may be responsible for this association.

\section{Case Report}

A 62-year-old woman presented with a worsening six-month history of progressively increasing dyspnea on exertion, cough with expectoration of blackish-brown mucus plugs and wheezing for the same duration. She denied history of hemoptysis, chest pain or fever. The patient was diagnosed as a case of pulmonary tuberculosis 40 years ago and received treatment with anti-tubercular drugs for one year. The diagnosis was based on sputum smear which was positive for acid-fast bacilli. The patient could not recollect her vaccination status, the results of a tuberculin skin test or the specific details of the drugs used; a sputum culture for mycobacteria was not performed during that period. Following the treatment, the patient was told she was cured of tuberculosis but she continued to experience dyspnea on strenuous activities but the symptoms had remained the same without any worsening but for the last six months. On examination, the patient was afebrile with a heart rate of 86 beats/minute, respiratory rate of 28 breaths/ minute and blood pressure of 128/76 mmHg. Examination of the respiratory system revealed normal breath sounds along with polyphonic wheezing as well as inspiratory and expiratory crackles in left infraclavicular area. General physical and remaining systemic examination was unremarkable. A chest radiograph showed fibrotic bands and volume loss in left upper zone while rest of the lung fields showed scattered alveolar opacities and cystic spaces (figure 1). High resolution computed tomography (HRCT) scan of thorax showed parenchymal fibrosis and volume loss in left upper lobe while central bronchiectasis, mosaic attenuation, small nodules (in a centrilobular distribution) and tree-in-bud pattern were observed in the other lobes (figures 2 and 3). Spirometry showed mild obstruction - forced expiratory volume in 1st second $\left(\mathrm{FEV}_{1}\right)$ of 0.98 liters and forced vital capacity (FVC) of 1.45 litres (values being 62.0\% and $61.5 \%$ of predicted values respectively) with the $\mathrm{FEV}_{1} / \mathrm{FVC}$ ratio being $67.6 \%$. 


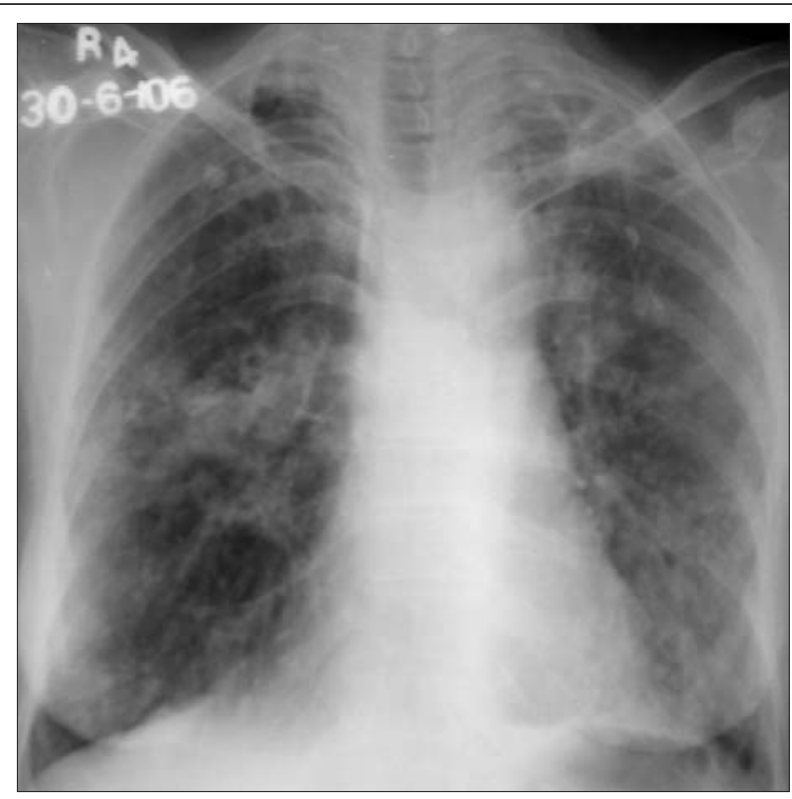

Fig. 1. - Chest radiograph showed linear opacities suggesting fibrotic bands in both the upper lobes and lower zones (tenting of diaphragm) while the right middle zone shows areas of non-homogenous alveolar opacities. Both the mid-zones also show numerous cystic spaces.

The post-bronchodilator values of $\mathrm{FEV}_{1}$ and FVC were 1.06 litres and 1.67 liters. Subsequently a work up for ABPA was carried out. Skin test for Aspergillus fumigatus (type 1 and type 3 hypersensitivity reactions) was positive. Serum precipitating antibodies against A fumigatus were present. The absolute eosinophil count was $1120 / \mu \mathrm{L}$, serum total $\mathrm{IgE}$ levels were $2200 \mathrm{IU} / \mathrm{ml}$ and specific IgE against $A$ fumigatus was $17.7 \mathrm{kUA} / \mathrm{L}$ (normal, less than $0.35 \mathrm{kUA} / \mathrm{L}$ ). A diagnosis of ABPA was thus made and patient initiated on oral prednisolone 0.75 milligram/kilogram body weight every day. The patient also received oral sustained-release theophylline at a dose of $450 \mathrm{mg} /$ day. On follow up after 6 weeks, she had significant improvement in her symptoms along with a reduction in IgE levels to less than $50 \%$ of baseline levels. Prednisolone was continued for a total duration of eight months and then terminated.

\section{Discussion}

The association between Mycobacterium tuberculosis and Aspergillus spp is not new. The latter is well known to colonize pre-existing lung cavities and produce fungal balls or aspergilloma. The majority of these cavities are a sequelae of PTB $[3,4]$. Moreover, ABPA is often misdiagnosed as PTB since patients can have similar clinical features and radiological manifestations [5]. In fact nearly one third to a half of the patients with ABPA may receive anti-tubercular therapy before the former is diagnosed $[6,7]$.

ABPA is known to occur in $1-2 \%$ of patients with asthma and in $6-25 \%$ of patients with cystic fibrosis [8, 9]. However, its prevalence in other patients with pre-existing structural lung disease is unknown. Occurrence of ABPA in patients previously treated for PTB is rare with just a single case

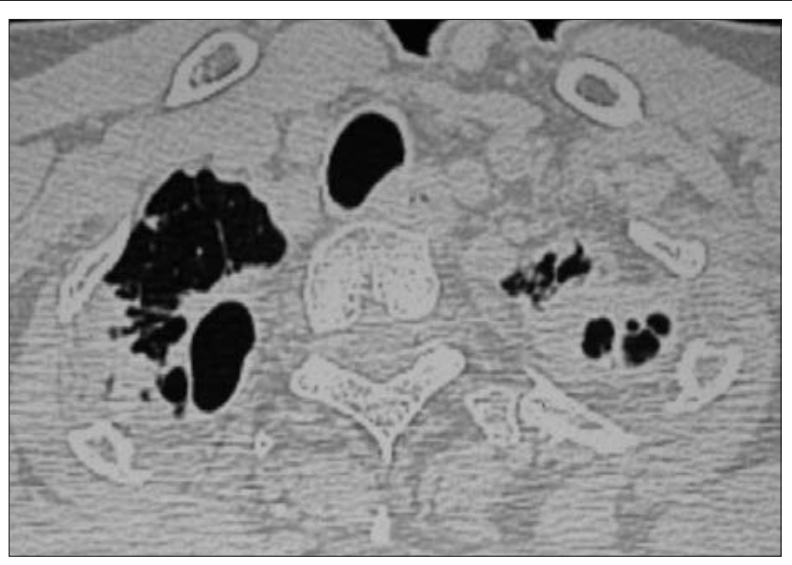

Fig. 2. - High-resolution computed tomography of the chest shows dense areas of fibrosis with numerous cavities in both the upper lobes.

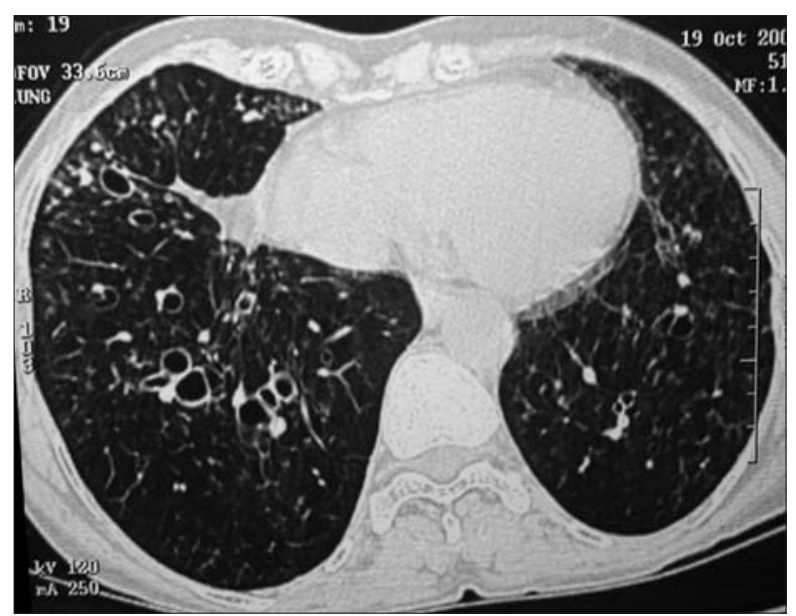

Fig. 3. - High-resolution computed tomography of the chest shows bilateral areas of bronchiectasis. In addition centrilobular nodules with tree-in-bud is also seen in the right lower lobe.

report [2]. The index patient was evaluated for ABPA in view of the history of expectoration of blackish brown mucus plugs - a symptom that occurs in nearly half of patients with ABPA [7]. It could also be debated whether the fibrosis and volume loss seen in left upper lobe were due to ABPA per se rather than as a sequelae of healed PTB. However, the fact that the patient had documented sputum positive pulmonary tuberculosis and the temporal profile of the patient suggests otherwise. It would also be distinctly unusual for a patient with active ABPA to have disease remission with anti-tubercular therapy alone and have the disease quiescent for almost 40 years without any therapy.

It is well known that patients with ABPA have a polarization of the immune response towards the CD4+/ Th2 type followed by production of cytokines like interleukin 4 (IL-4), interleukin 5 (IL-5) and interleukin 13 (IL-13) [10]. Enhanced susceptibility of circulating B cells to IL-4 in particular serves to initiate and sustain the elevation in serum levels of IgE (total and Aspergillus specific), a characteristic feature of ABPA. This polarization of the immune response and recruitment of CD4+/ Th2 lymphocytes specific for Aspergillus fumigatus to the bronchi and bronchioles that contain mucus 
plugs impacted with hyphae of $A$. fumigatus is believed to be mediated by chemokines especially interleukin-8 (IL-8) [10]. In contrast, the protective immune response against tuberculosis is of the CD4+/ Th1 type $[11,12]$. Development of cell mediated immunity and production of interferon $\gamma$ (IFN- $\gamma$ ) are essential for control of the disease. Toll like receptors (TLRs) especially TLR-2 are believed to mediate the recognition of mycobacterial antigens by macrophages and dendritic cells. Production of cytokines like tumour necrosis factor $\alpha$ (TNF- $\alpha$ ) and IFN- $\gamma$ by activated macrophages and CD4+ lymphocytes is associated with formation and maintenance of the granuloma, a critical step in the development of immunity against Mycobacterium tuberculosis and thus control of the disease [13]. It is therefore not surprising that exposure to $M$. $t u$ berculosis has been associated with a reduced risk of developing asthma $[14,15]$. However, once TB has been treated, the Th1 response decreases and there is a relative preponderance of $\mathrm{Th} 2$ response which can increase the susceptibility of bronchial asthma [16] and probably ABPA. Whether patients with cured PTB have increased Th2 response compared to normal controls is not known.

The intriguing point therefore remains as to whether there was any correlation between the occurrence of ABPA and PTB or was it just a co-incidence. It can be argued that the disease in the upper lobes was as a consequence of pulmonary tuberculosis which was followed by colonisation with Aspergillus and evolved in to ABPA. Two questions which still remain unanswered are: (a) why do all the pulmonary tuberculosis scar patients not develop ABPA after completion of anti-tubercular treatment? And, whether the Th1 response is retained, with the possible presence of little inactive $M$. tuberculosis even after treatment?; and, (b) whether familial susceptibility to ABPA may be the answer as several host factors including HLA-DR2 polymorphisms, IL-10 polymorphisms, surfactant protein A2 and CFTR (cystic fibrosis transmembrane receptor) polymorphisms have been implicated in pathogenesis of ABPA [17]. An increase in the description of cases with similar coexistence in the future remains a possibility in view of the increasing recognition of ABPA [7].

Although it has not ben performed in this case, sputum analysis may be useful in monitoring the course of ABPA, and direct measures of sputum inflammation may be a more sensitive predictor of disease activity. In one study, the sputum eosinophil and neutrophil numbers, and sputum eosinophil cationic protein levels were markedly elevated in patients with ABPA with concomitant bronchiectasis than those without. The sputum cellular count also correlated closely with the severity of bronchiectasis on HRCT [18].

Finally, the limitation of this report is obvious because of the single case reported. The current case only suggests the hypothesis of a possible association between previous and cured TB with ABPA. The issue can only be resolved by a welldesigned epidemiological study as the observation of one single case of such association does not bring additional evidence on the existence of excess risk of ABPA among cured TB patients.

In conclusion, current criteria require the presence of asthma or cystic fibrosis for recognition of ABPA. However, patients with TB scars can also develop ABPA during the course of their disease. Larger observations are required to evaluate this association.

\section{References}

1. Eppinger TM, Greenberger PA, White DA, Brown AE, Cunningham-Rundles C. Sensitization to Aspergillus species in the congenital neutrophil disorders chronic granulomatous disease and hyper-IgE syndrome. $J \mathrm{Al}$ lergy Clin Immunol 1999; 104: 1265-72.

2. Yanase K, Nakamura M, Toyoda T, et al. A case of allergic bronchopulmonary aspergillosis following active pulmonary tuberculosis. Arerugi 1996; 45: 1181-4.

3. Caidi M, Kabiri H, Al Aziz S, El Maslout A, Benosman A. Surgical treatment of pulmonary aspergilloma. 278 cases. Presse Med 2006; 35: 1819-24.

4. Regnard JF, Icard P, Nicolosi M, et al. Aspergilloma: a series of 89 surgical cases. Ann Thorac Surg 2000; 69: 898-903.

5. Al-Moudi OS. Allergic bronchopulmonary aspergillosis mimicking pulmonary Tuberculosis. Saudi Med J 2001; 22: 708-13.

6. Behera D, Guleria R, Jindal SK, Chakrabarti A, Panigrahi D. Allergic bronchopulmonary aspergillosis: a retrospective study of 35 cases. Indian J Chest Dis Allied Sci 1994; 36: 173-9.

7. Agarwal R, Gupta D, Agarwal AN, Behera D, Jindal SK. Allergic bronchopulmonary aspergillosis: lessons from 126 patients attending a chest clinic in north India. Chest 2006; 130: 442-8.

8. Greenberger PA. Allergic bronchopulmonary aspergillosis. J Allergy Clin Immunol 2002; 110: 685-92.

9. de Almeida MB, Bussamra MH, Rodrigues JC. Allergic bronchopulmonary aspergillosis in paediatric cystic fibrosis patients. Paediatr Respir Rev 2006; 7: 67-72.

10. Moss RB. Pathophysiology and immunology of allergic bronchopulmonary aspergillosis. Med Mycol 2005; 43 (Suppl 1): S203-6.

11. Tan YJ, Zhang YB, Feng DY, et al. The change and the clinical significance of peripheral blood Th1/Th2 cells in patients with pulmonary tuberculosis. Zhonghua Jie He He Hu Xi Za Zhi 2004; 27: 385-9.

12. Lienhardt C, Azzurri A, Amedei A, et al. Active tuberculosis in Africa is associated with reduced Th1 and increased Th2 activity in vivo. Eur J Immunol 2002; 32: 1605-13.

13. Ferraz JC, Melo FB, Albuquerque MF, Montenegro SM, Abath FG. Immune factors and immunoregulation in tuberculosis. Braz J Med Biol Res 2006; 39: 1387-97.

14. von Mutius E, Pearce N, Beasley R, et al. International patterns of tuberculosis and the prevalence of symptoms of asthma, rhinitis, and eczema. Thorax 2000; 55: 449-53.

15. Jones PD, Gibson PG, Henry RL. The prevalence of asthma appears to be inversely related to the incidence of typhoid and tuberculosis: hypothesis to explain the variation in asthma prevalence around the world. Med Hypotheses 2000; 55: 40-2.

16. Rajasekaran S, Savithri S, Jeyaganesh D. Post-tuberculosis bronchial asthma. Ind J Tub 2001; 48: 139-42.

17. Gibson PG. Allergic Bronchopulmonary Aspergillosis. Semin Respir Crit Care Med 2006; 27: 185-91.

18. Wark PA, Saltos N, Simpson J, Slater S, Hensley MJ, Gibson PG. Induced sputum eosinophils and neutrophils and bronchiectasis severity in allergic bronchopulmonary aspergillosis. Eur Respir J 2000; 16: 1095-101. 